\title{
A NOVEL EXPERIMENTAL NOISE POWER RATIO CHARACTERIZATION METHOD FOR MULTICARRIER MICROWAVE POWER AMPLIFIERS
}

\author{
T. Reveyrand", D. Barataud", J. Lajoinie", M. Campovecchio", \\ J.-M. Nebus", E.Ngoya", J.Sombrin", D. Roques" \\ IRCOM - UMR n6615 - 123, Av. Albert Thomas, 87060 LIMOGES Cedex (France), \\ "CNES - 18, Av. E. Belin, 31401 TOULOUSE Cedex (France), \\ ".'ALCATEL SPACE INDUSTRIES - 26, Av. Jean-Francois Champollion, BP 1187, 31037 TOULOUSE Cedex (France)
}

\begin{abstract}
The linearity characterization of multicarrier power amplifiers in terms of Noise Power Ratio (NPR) is now well adopted. The use of an analog band pass noise source with a notch in the center bandwidth is currently used for this purpose. We present in this paper a novel NPR measurement technique based on a numerical generation of a gaussian noise. This experimental noise generation is similar to the technique used in non linear circuit level or system level simulations using the envelope transient technique. The measurement principle is based on the use of a baseband arbitrary waveform generator $(A W G)$ and an upconverter chain from baseband to $L$ or $S$ band. The large signal Noise stimuli drive the amplifier under test. The output signal of the amplifier is downconverted back to baseband and the resulting complex envelope is recorded with a sampling scope and computed for NPR extraction. A comparison of NPR measurements obtained with our method and with the conventional analog noise source technique is presented. Other possible applications of our measurement system are also mentioned and emphasize the capabilities of the proposed characterization tool.
\end{abstract}

\section{INTRODUCTION}

The linearity of multicarrier power amplifiers cannot be accurately characterized in terms of third order intermodulation by using two tone test signals. Complex phenomena like nonlinear memory effects implie that in depth linearity characterization of power amplifiers must be performed with specific test signals representative of the complex modulated signals encountered in real applications.

Gaussian bandlimited noise is now widely adopted for this purpose and provides a linearity criterion known as NPR [1]. Conventional NPR measurement systems are based on the use of an analog white noise source as depicted in figure 1 [2][3].

The noise supplied by this source is bandlimited using a bandpass filter. A notch filter is used to reject a little part of the noise around the center bandwidth. The resulting signal is upconverted to the microwave domain and linearly amplified to drive the Amplifier Under Test (AUT). Due to the nonlinear behavior of the AUT, the notch of the output signal spectrum is partially filled. The measurement with a spectrum analyzer of the ratio between the mean power out of the notch and within the notch provides the NPR.

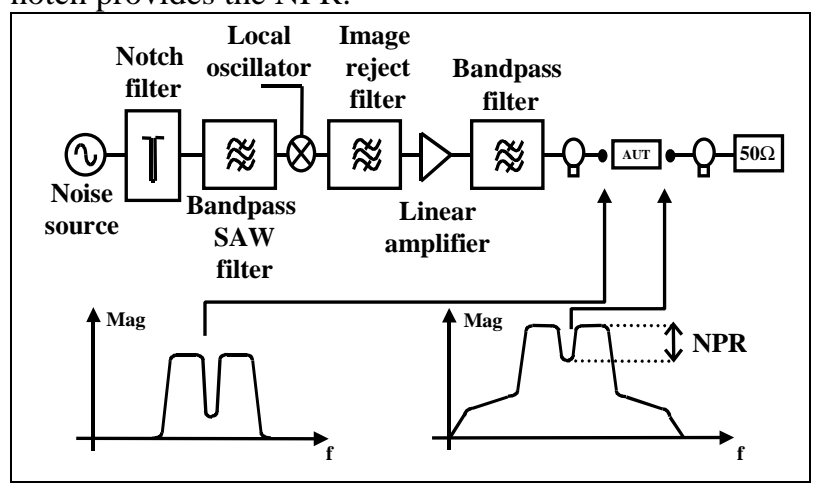

Fig. 1. : Conventionnal NPR characterization technique.

The novel approach for NPR measurements proposed in this paper is based on a numerical generation of a band pass gaussian noise by using a baseband arbitrary waveform generator (AWG).

A computer controlled AWG is used to synthesize a great number of CW tones (1000 to 10000) with equal magnitudes, different frequencies and random phases in the frequency bandwidth (DC $-100 \mathrm{MHz}$ ).

As long as the number of carriers is large enough, and according to the central limit theorem, the resulting multicarrier signal is a band pass gaussian noise. A great advantage offered by this technique is to make easy the suppression of few tones ( 1 to 10 percent 
of the total number) anywhere within the frequency bandwidth. Therefore, an ideal notch can be created to build an appropriate signal feeding the AUT and to measure the NPR.

The baseband noise is upconverted to the microwave domain by using an I/Q modulator. The output RF signal of the AUT is down converted back to the baseband by using an I/Q demodulator, measured with a sampling scope and processed to compute NPR.

In the following parts of the paper, the application of our characterization tool to NPR measurements is described. Nevertheless, the linearity characterization of power amplifiers submitted to any kind of digital modulation multiplex can be achieved. This is an important advantage of our characterization approach compared to the analog noise source technique.

Trade-offs between linearity and power added efficiency of power amplifiers submitted to any kind of modern communication signals (like OFDM, CDMA, coherent pulsed FM/CW) can be investigated.

\section{THE PROPOSED NPR CHARACTERIZATION PRINCIPLE}

The proposed NPR characterization method is based on the generation of a multi $\mathrm{CW}$-carrier signal to feed the input of any amplifier under test [4][5]. Basically, this signal is composed of 1000 to 10000 simultaneous $\mathrm{CW}$ carriers with equal magnitudes and random phases (uniform distribution on $0-2 \pi$ ).

The carriers are regularly spaced within a channel bandwidth and few carriers (5 to $10 \%$ ) are removed from the center bandwidth to create an ideal notch as illustrated in figure 2 .

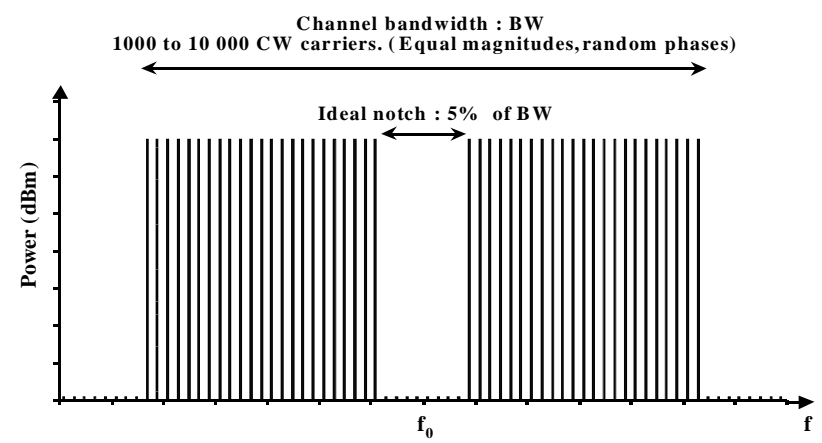

Fig. 2. : Multi CW carriers for the synthesis of noise stimuli.

The resulting signal can be written as following :
$x(t)=\sum_{n=1}^{\frac{N}{2}-\frac{M}{2}} A \cos \left(2 \pi f_{n} t+\varphi_{n}\right)+\sum_{n=\frac{N}{2}+\frac{M}{2}}^{N} A \cos \left(2 \pi f_{n} t+\varphi_{n}\right)$

(1)

(N-M) : number of carriers (1000 to 10000)

$\mathrm{M}$ : number of rejected carriers

$\frac{M}{N}: 5$ to $10 \%$

$\varphi_{n}$ : uniform distribution on $[0-2 \pi]$

As long as the number of carriers is large and according to the central limit theorem, $x(t)$ is a band limited gaussian noise.

A time domain representation of $x(t)$ and the statistic distribution of the magnitude of $x(t)$ are given in figure 3 .
Time Domain NPR Signal

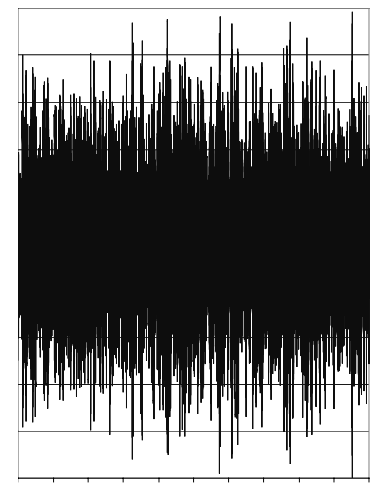

Magnitude Distribution of the Baseband Signal

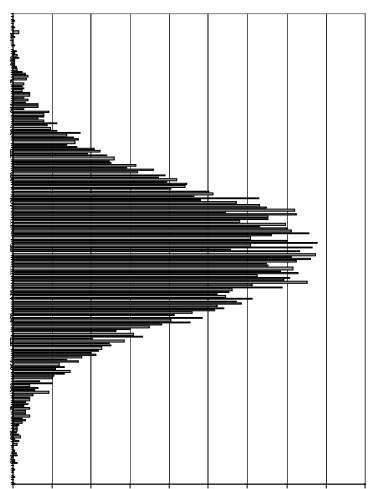

Fig. 3. : Time domain record of $1000 \mathrm{CW}$ carriers with random phases.

When $\mathrm{x}(\mathrm{t})$ feeds a non linear amplifier, the spectrum of the output signal looks like the one depicted in figure 4 . Multiple intermodulation combinations partially fill the notch and the output signal $\mathrm{y}(\mathrm{t})$ can be written as following if we omit spurious out of the channel bandwidth :

$y(t)=\sum_{n=1}^{\frac{N}{2}-\frac{M}{2}} A_{n} \cos \left(2 \pi f_{n} t+\theta_{n}\right)+\sum_{\frac{N}{2}+\frac{M}{2}}^{N} A_{n} \cos \left(2 \pi f_{n} t+\theta_{n}\right)$ 
Then, the NPR is defined by:

$$
N P R=\frac{\left\{\sum_{n=1}^{\frac{N}{2}-\frac{M}{2}} \frac{A_{n}^{2}}{2}+\sum_{n=\frac{N}{2}+\frac{M}{2}}^{N} \frac{A_{n}^{2}}{2}\right\} \frac{1}{N-M}}{\left\{\sum_{n=\frac{N}{2}-\frac{M}{2}}^{\frac{N}{2}+\frac{M}{2}} \frac{A_{n}^{2}}{2}\right\} \frac{1}{M}}
$$

(3)

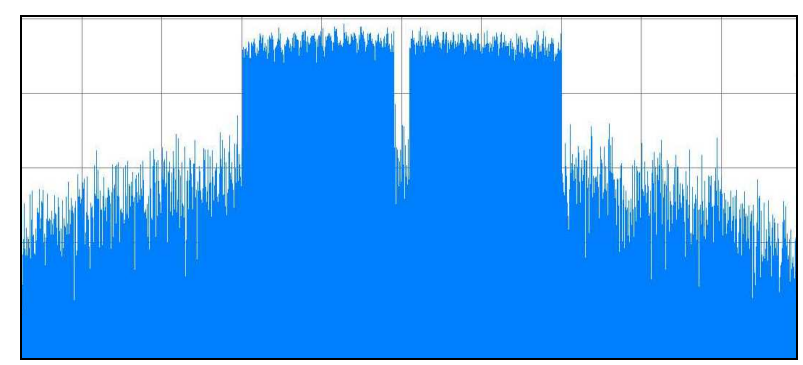

Fig. 4. : Typical spectrum of the output signal of a non linear amplifier.

\section{MEASUREMENT SYSTEM.}

The block diagram of the measurement system is given in figure 5. The synthesis of a baseband multicarrier signal (typically DC $-60 \mathrm{MHz}$ ) with a notch is performed by using a computer controlled arbitrary waveform generator (AWG 2021 Tektronix, 12 bits $250 \mathrm{MHz}$ sampling rate).

This baseband signal is upconverted to $\mathrm{L}$ or $\mathrm{S}$ band using an I/Q modulator. The resulting signal is linearly amplified and drive the input of the Amplifier Under Test.

The output signal of the AUT feeds an I/Q demodulator and baseband output signals are measured by using a sampling scope (TDS 500 Tektronix -8 bits - $1 \mathrm{Gs} / \mathrm{s})$. A FFT is performed to compute NPR as defined in expression (3).

A computer controlled step attenuator is connected at the input of the I/Q demodulator to keep this device operating in its linear region

A tuner is connected to the AUT output for investigations on trade-off between NPR and power added efficiency versus load impedance.

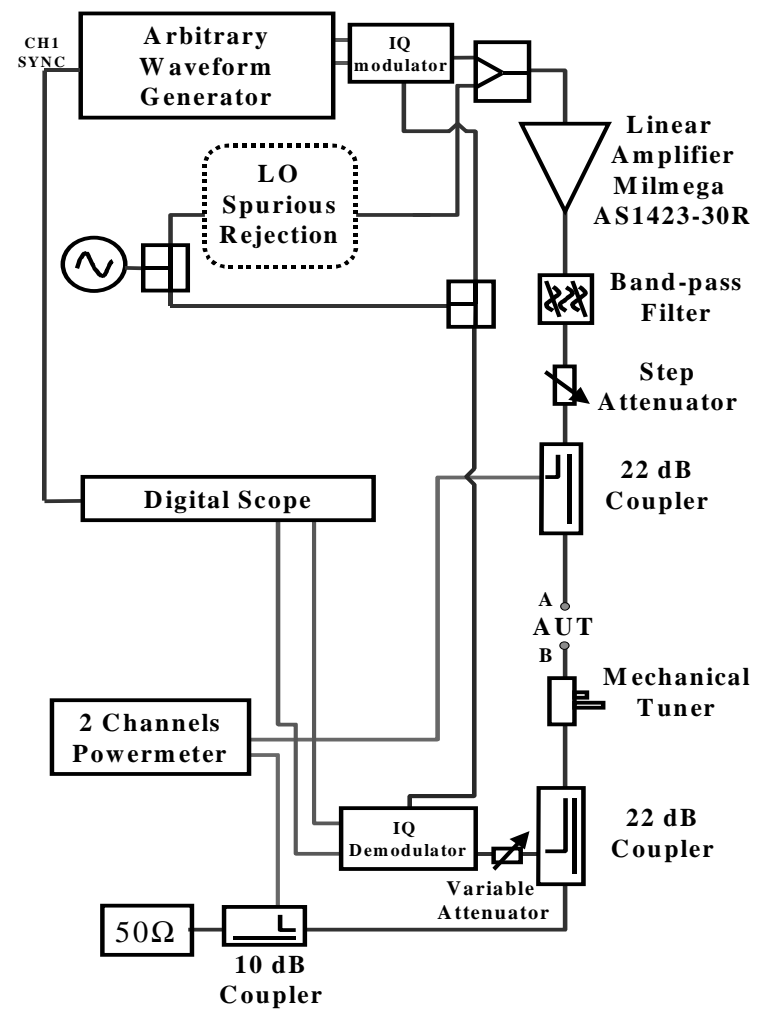

Fig. 5. : The characterization block diagram.

\section{MEASUREMENT RESULTS}

NPR measurements of a $23 \mathrm{dBm}$ TI-HFET amplifier were performed at $2.18 \mathrm{GHz}(20 \mathrm{MHz}$ bandwidth). Envelope transient technique applied to NPR calculations (as explained in [6]), was used to design this amplifier for an optimized trade-off between PAE and NPR. Figure 6 shows NPR measurements obtained with 1000 regulary spaced $\mathrm{CW}$ frequencies in the $20 \mathrm{MHz}$ bandwidth. Each curve differs from the random phase outcome used. $3 \mathrm{~dB}$ NPR variations depending on the phase outcome are observed. The conclusion is that 1000 carriers are not sufficient for an accurate characterisation of NPR.

In fact, at each fixed input average power, the peak to average value differs from one curve to another, depending on the phase outcome chosen. Therefore the non linearity of the AUT is differently excited and the NPR is different. 


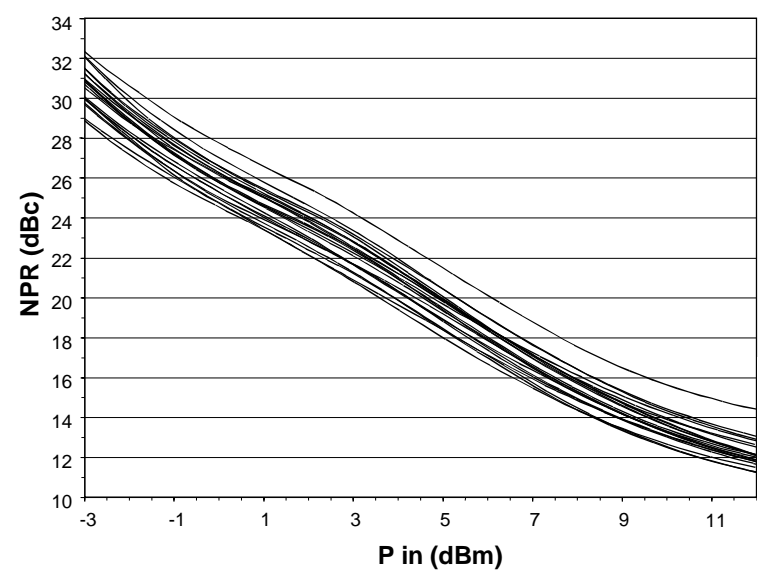

Fig. 6. : NPR vs $\mathrm{P}$ in $(1000 \mathrm{CW})$

If we use now $10000 \mathrm{CW}$ carriers to generate baseband gaussian noise, there is no difference in NPR measurements when the random phase outcome of those 10000 carriers is changed, as shown in figure 7.

Moreover, NPR measurements are in good agreement with NPR measurements performed with the conventional analog noise source technique. Such measurements demonstrate that a great number of carriers are required for an accurate NPR characterization of power amplifiers. NPR predictions by simulations using this same approach requires also a great number of carriers $(10000)$. If a lower number of carriers is used (i.e. 1000) to reduce simulation time, it is absolutely necessary to compute an average of the results obtained from different random phase out comes.

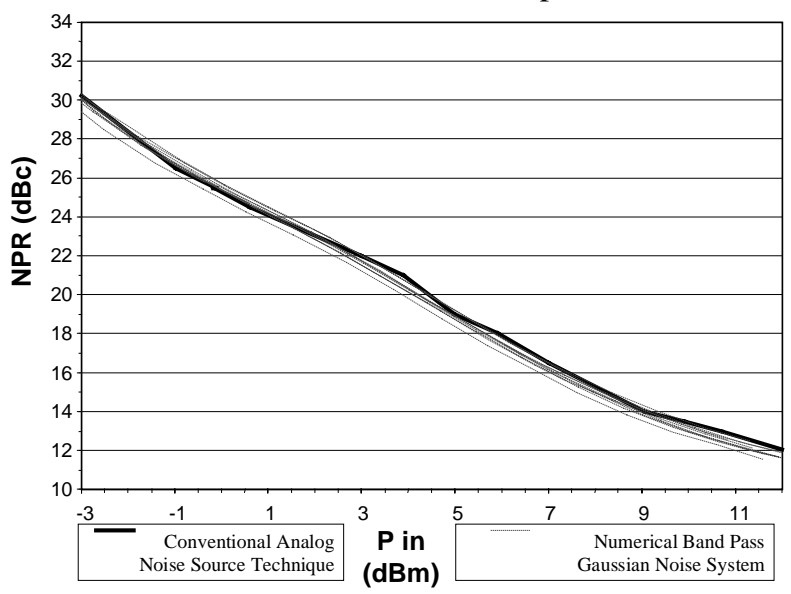

Fig. 7. : NPR vs $\mathrm{P}$ in (50 Ohms)

Figure 8, 9 and 10 show NPR, multicarrier output power and power added efficiency obtained when the load impedance of the TI HFET amplifier is pulled away from $50 \mathrm{Ohms}$ by means of a mechanical tuner.
For a given NPR (for example $16 \mathrm{dBc}$ ) a better output power can be reached if the output of the amplifier is mismatched. Nevertheless the best trade-off between NPR and PAE is obtained for a 50 ohms load as shown figure 10 . This proves that the internal matching circuit of this amplifier was well design for an optimum trade off between linearity and DC consumption.
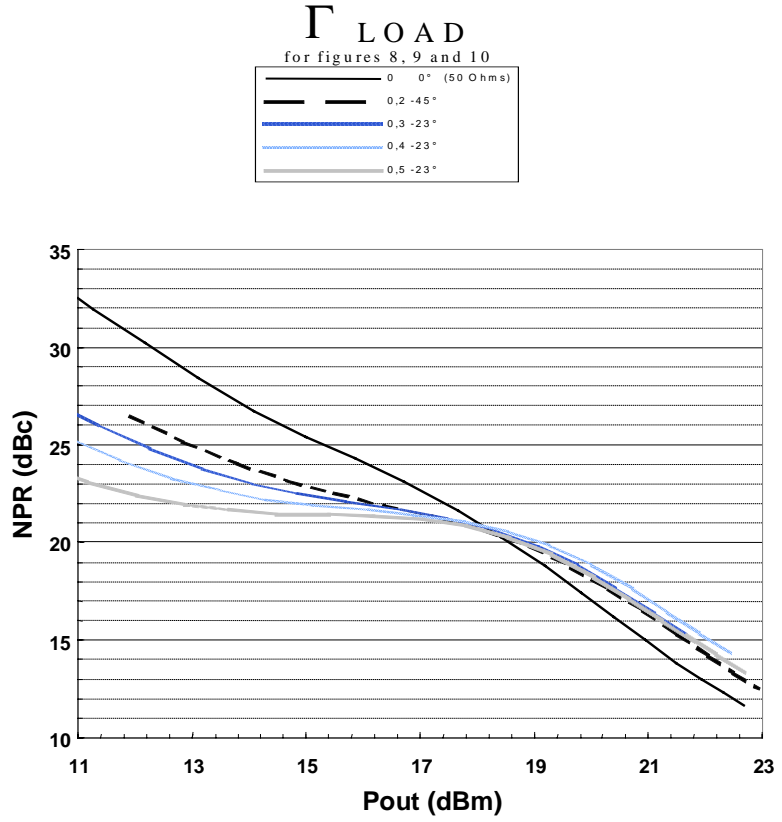

Fig. 8. : NPR vs P out

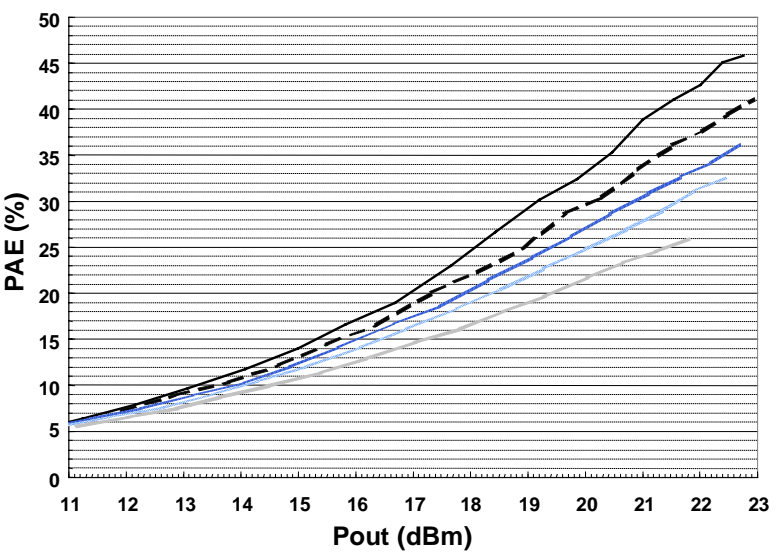

Fig. 9. : PAE vs P out 


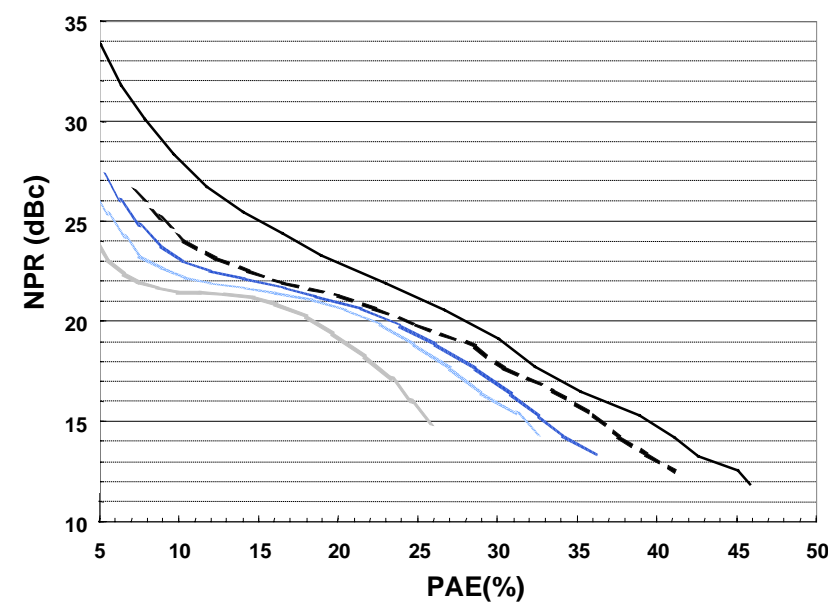

Fig. 10. : NPR vs PAE

\section{CONCLUSION}

A novel approach for the linearity characterization of power amplifiers in terms of NPR has been proposed. It provides valuable information in the sense that this method is based on the numerical generation of a gaussian noise that exactly corresponds to the NPR simulations using the envelope transient technique. Moreover this approach is not limited to NPR measurements as compared to the analog noise source technique. It can be used for the optimization of tradeoffs between linearity and efficiency of power amplifiers submitted to any kind of digital modulation.

Furthermore, such a characterization tool is very useful :

- to investigate on non linear memory effects when amplifiers are driven by complex modulated signals [7] ;

- to validate non linear electrical models of transistors taking into account low frequency time constants (thermal effects, trapping effects) [8].

An important contribution in the field of non linear black box modeling is also expected and under development.

Investigations on linearization of amplifiers can also be performed as baseband signals can be computed and/or predistorded using the computer controlled AWG [9].

\section{ACKNOWLEDGMENTS}

The authors would like to thank the French Space Agency (CNES) for their financial support, and F. Brasseau and O.Havard from Alcatel Space Industries (France) for NPR measurements using the analog white noise source technique.

\section{REFERENCES}

[1] A. Platzker, S. Bouthillette "Variable Output, High EfficiencyLow Distortion S-Band Power Amplifiers and Their Performances Under Single Tone and Noise Power Excitations", IEEE MTT-S Digest, 1995, pp.441-444.

[2] Noise/Com Incorporation "Test Set Speeds NPR Measurements", Microwaves \& RF, January 1994, pp.126-128.

[3] F. Brasseau, L. Chapus, G. Michaud, A. Darbandi "Réalisation d'un banc de mesures NPR large bande", JNM Conference Digest, 1997, pp.454-455.

[4] R.J. Westcott "Investigation of multiple f.m./f.d.m. carrieirs through a satellite t.w.t. operating near to saturation", Proc. IEE, Vol 114, No 6, June 1967, pp.726-740.

[5] Nuno Borges Carvalho and José Carlos Pedro "Multi-Tone Intermodulation Distortion Performance of $3^{\text {rd }}$ Order Microwave Circuits", IEEE MTT-S Digest, 1999, pp.763-766.

[6] J. Lajoine, E. Ngoya, D. Barataud, J.-M. Nebus, J. Sombrin, B. Rivierre "Efficient simulation of NPR for the optimum design of satellite transponders SSPAs.", IEEE MTT-S Digest, 1999, pp.741-744.

[7] Kaoru ISHIDA, Hikaru IKEDA, Hiroaki KOSUGI, Masaaki NISHIJIMA and Tomoki "A High Efficiency and Low Distortion GAAS Power MMIC Design in the Wide Load Impedance Range by Extended Use of Load-Pull Method", IEEE MTT-S Digest, 1999, pp.775-778.

[8] Th. Peyretaillade, M. Perez, Ph. Auxeméry, J.C. Lalaurie, R. Quéré "A Pulsed-Measurement Based Electrothermal Model of HBT with Thermal Stability Prediction Capalities", IEEE MTTS Digest, 1997, pp. 1515-1518.

[9] Ernesto G. Jeckeln, Fadhel M. Ghannouchi and Mohamad Sawan "Adaptative Digital Predistorter for Power Amplifiers with Real Time Modeling of Memoryless Complex Gains ", IEEE MTT-S Digest, 1996, pp.835-838. 